Global Mental Health

cambridge.org/gmh

\section{Etiology \\ Original Research Paper}

Cite this article: Ambaw F, Mayston R, Hanlon C, Alem A (2020). Incidence of depression in people with newly diagnosed tuberculosis in Ethiopia: a cohort study. Global Mental Health 7, e1, 1-9. https://doi.org/10.1017/gmh.2019.27

Received: 28 December 2018

Revised: 17 September 2019

Accepted: 13 November 2019

\section{Key words:}

Depression; Ethiopia; incidence; primary care; tuberculosis

\section{Author for correspondence:}

Fentie Ambaw, E-mail: fentiea.getahun@gmail. com

\title{
Incidence of depression in people with newly diagnosed tuberculosis in Ethiopia: a cohort study
}

Fentie Ambaw 1,2 (D), Rosie Mayston ${ }^{3}$, Charlotte Hanlon ${ }^{2,3,4}$ and Atalay Alem²

${ }^{1}$ Bahir Dar University, School of Public Health, Bahir Dar, Ethiopia; ${ }^{2}$ Department of Psychiatry, Addis Ababa University, College of Health Sciences, School of Medicine, Addis Ababa, Ethiopia; ${ }^{3}$ King's College London, Institute of Psychiatry, Psychology and Neuroscience, Centre for Global Mental Health, London, UK and ${ }^{4}$ Centre for Innovative Drug Development and Therapeutic Trials for Africa (CDT-Africa), College of Health Sciences, Addis Ababa University, Addis Ababa, Ethiopia

\begin{abstract}
Background. Cross-sectional studies show that the prevalence of comorbid depression in people with tuberculosis (TB) is high. The hypothesis that TB may lead to depression has not been well studied. Our objectives were to determine the incidence and predictors of probable depression in a prospective cohort of people with TB in primary care settings in Ethiopia. Methods. We assessed 648 people with newly diagnosed TB for probable depression using Patient Health Questionnaire, nine-item (PHQ-9) at the time of starting their anti-TB medication. We defined PHQ-9 scores 10 and above as probable depression. Participants without baseline probable depression were assessed at 2 and 6 months to measure incidence of depression. Incidence rates per 1000-person months were calculated. Predictors of incident depression were identified using Poisson regression.

Results. Two hundred and ninety-nine (46.1\%) of the participants did not have probable depression at baseline. Twenty-two (7.4\%) and $26(8.7 \%)$ developed depression at 2 and 6 months of follow up. The incidence rate of depression between baseline and 2 months was 73.6 (95\% CI 42.8-104.3) and between baseline and 6 months was 24.2 (95\% CI 14.9-33.5) per 1000 person-months respectively. Female sex (adjusted $\beta=0.22$; 95\% CI $0.16-0.27$ ) was a risk factor and perceived social support (adjusted $\beta=-0.14 ; 95 \% \mathrm{CI}-0.24$ to -0.03 ) was a protective factor for depression onset.

Conclusion. There was high incidence of probable depression in people undergoing treatment for newly diagnosed TB. The persistence and incidence of depression beyond 6 months need to be studied. TB treatment guidelines should have mental health component.
\end{abstract}

\section{Introduction}

Cross-sectional studies in both high income (Trenton and Currier, 2001) and low and middle income countries (Mathai et al., 1981; Aydin and Ulusahin, 2001; Moussas et al., 2008; Issa et al., 2009; Sulehri et al., 2010; Ige and Lasebikan, 2011; Panchal, 2011; Doherty et al., 2013; Duko et al., 2015) show that comorbid depression among people with TB is a common debilitating condition with a prevalence of as high as 50.0\% (Sweetland et al., 2018). Recently, we have analyzed the prevalence of probable depression in people newly diagnosed with tuberculosis (TB) at the time of initiation of their anti-TB medication in primary care settings in Ethiopia and found that it was $54.0 \%$ on nine-item version of Patient Health Questionnaire (PHQ-9) at a cut-off point of 10 and above (Ambaw et al., 2017).

Attempts to understand the type of relationship between TB and depression are limited. A number of explanations have been given in the literature (Sweetland et al., 2017). Some evidence suggests that $\mathrm{TB}$ and depression may share risk factors (Kiecolt-Glaser and Glaser, 2002; Reiche et al., 2004; Katon et al., 2007). Others explain that TB can be contracted as a result of compromised immunity and neglected self-care associated with depression (Reiche et al., 2004). Such evidence shows that depression enhances the production of proinflammatory cytokines and directly minimizes immunological competence by down regulating cellular and humoral responses (Kiecolt-Glaser and Glaser, 2002; Reiche et al., 2004; Katon et al., 2007; Katon, 2011). The third hypothesis, which is also the focus of this study, is that people with TB may develop depression through various mechanisms including chronic infection and related disability and psycho-socioeconomic stressors (Mikkelsen et al., 2004), effects of TB medications such as isoniazid (Madan et al., 1989), chronic infectious conditions which may lead to overproduction of proinflammatory cytokines such as interleukin 6 , which facilitate cascades of endocrine reactions that are suggested to result in depressive symptoms (Kiecolt-Glaser and Glaser, 2002), and general physical and psychological losses (Mikkelsen et al., 2004; Pachi et al., 2013). However, whether people with a newly diagnosed TB receiving anti-TB 
medications will develop new depressive disorder or not has not been properly studied. A study in Taiwan followed people with TB for a mean period of 6.5 years and reported that they had a higher incidence of depression compared to controls. This study has not adequately controlled socioeconomic factors and has included only cases of pulmonary TB (Yen et al., 2015).

Understanding whether incident depression occurs in people with TB after starting anti-TB care is critically required to improve the national guideline for clinical and programmatic management of TB in Ethiopia and similar settings that lack mental health component (Federal Ministry of Health of Ethiopia, April 2012), and to target depression interventions in the integration of mental health care into primary care which is being scaled up in Ethiopia (FMOH, 2012a, 2012b). It also provides a strong base to conduct well designed studies on the relationship between depression and anti-TB medications, specifically isoniazid (Doherty et al., 2013) and ethambutol (Yen et al., 2015), TB-related stigma or behavioral factors specific to this population (Sweetland et al., 2018).

The objectives of this study were to assess incidence rates of probable depression at 2 and 6 months after starting anti-TB treatment and to identify factors predicting incidence in the context of TB and its treatment.

\section{Methods}

Design

This study was part of a 6 month prospective observational cohort that examined the interaction between depression and newly diagnosed TB in primary care settings in Ethiopia (Ambaw et al., 2015). At the time of diagnosis and treatment initiation for TB, we assessed for depression and we classified participants into 'having probable depression' and 'not having probable depression' based on PHQ-9, scores. We then followed those 'not having probable depression' for incidence of probable depression during the 6 month follow up period.

\section{Study setting}

The study was conducted from December 2014 to July 2016 in 14 primary care centers located in south central (i.e. in Silti and Gurage zones) and northern (i.e. Bahir Dar zone) Ethiopia. Two of the primary care centers were hospitals and 12 were health centers. Similar services were provided at the health centers and hospitals: providers of TB care were nurses or public health officers that have taken the same trainings on TB care, the same medications were given at both institutions, and the same treatment guidelines were used. All people with newly diagnosed TB in the outpatient departments were being treated according to the Directly Observed Treatment, Short course (DOTS) regimen. DOTS has 6 months duration with intensive and continuation phases. The intensive phase consists of treatment with combination of four medications (rifampicin, ethambutol, isoniazid, and pyrazinamide) for the first 2 months, and the continuation phase consists of a combination of two medications (rifampicin and isoniazid), to be taken for 4 months immediately after the intensive phase (Federal Ministry of Health of Ethiopia, April 2012).

The frontline nurses and public health officers working in those primary care centers received training in the management of mental disorders according to the evidence-based WHO
Mental health Gap Action Programme Intervention Guide (mhGAP-IG) for mental, neurological, and substance use disorders in non-specialized health care settings (WHO, 2016). The presence of staff that took this training and TB patient flow of at least five per month were criteria to include the primary care centers in the study areas.

\section{Eligibility criteria}

- People attending the selected health centers for TB treatment who were within 1 month of starting anti-TB treatment

- Aged 18 years and above

- No plan to move out of the study area

- Not too ill to be interviewed at baseline as perceived by the interviewer or the prospective participant

- Had not been admitted to an in-patient unit for more than 5 days in the last 1 month as the additional stressors of being hospitalized represent a different range of risk factors for depression.

- Not diagnosed with Multidrug-Resistant Tuberculosis (MDRTB); people with MDR-TB constitute a different population because their treatment and outcomes are different (medications with a higher side effect burden and taken for a much longer duration; poorer prognosis) and MDR-TB is amore feared and stigmatized condition (Vega et al., 2004). Furthermore, only one of the study health facilities had recently started a service for people with MDR-TB.

- Not on re-treatment for TB as people who experienced previous treatment failures are at high risk of MDR-TB and constitute a different risk group for depression.

\section{Sample size}

The total sample size was 648 . The sample size was based on the primary objective of the planned longitudinal study which was to examine the effect of depression on default from anti-TB treatment (Ambaw et al., 2015) and was calculated using the following parameters: $80 \%$ power, $95 \%$ confidence level, $2.5 \%$ prevalence of treatment default among patients with TB and without depression, $7.5 \%$ prevalence of treatment default among people with $\mathrm{TB}$ and co-morbid depression and a ratio of 2:1 of non-exposed (not depressed) to exposed (depressed) participants. This provided a required sample size of 639 . With a contingency of $10 \%$ for possible loss to follow up, the target sample was 703 people with TB.

\section{Variables and measurements}

\section{Dependent variable: probable depression}

Depression was measured using the PHQ-9. The PHQ-9 assesses the presence of nine depressive symptoms over the past 2 weeks in an individual; the possible responses are: not at all (0), several days (1), more than half of the days (2) and almost every day (3); possible composite score ranges from zero to 27. Globally, the scale is widely used in surveys, effectiveness trials and cohort studies in various populations (Kroenke et al., 2010). In Ethiopia, it has been validated two times and was found to be useful in screening depression in adult out patients (Gelaye et al., 2013; Hanlon et al., 2015). The optimum cut-off point was five and above in primary healthcare centers in a rural district (Hanlon et al., 2015) and 10 and above in outpatient medical clinics in a referral hospital in Addis Ababa (Gelaye et al., 2013). We applied 
the more conservative cut-off point of 10 and above to define probable depression. In the baseline data of this study, the PHQ-9 had a single dimension structure, a Cronbach's $\alpha$ value of 0.81 and a mean inter-item correlation coefficient of 0.33 (Ambaw et al., 2017). In this manuscript, the term depression is used instead of probable depression for simplicity purpose.

\section{Independent variables}

(I) Socio-demographic variables: age, sex, marital status, level of education, religion, household income, occupation and place of residence (urban $v$. rural) were measured by self-report. Household income was measured by asking the participants to estimate the monthly total income of their household. When the participant was a farmer, we changed the estimates of annual income in kind to cash using the local market price. We converted the monthly income into annual income.

(II) Perceived social support: perceived social support was measured using the three-Item Oslo Scale of Perceived Social Support (OSLO-3) with scores ranging from 3-14 (Meltzer, 2003). The scale was previously reported to work well in TB patients in Ethiopia (Duko et al., 2015). In this sample the scale had an $\alpha$ value of 0.61 and a mean interitem correlation of 0.35 . Higher scores on OSLO-3 show better perceived social support.

(III) TB-related stigma: TB related stigma was measured at the second assessment using a 10-item TB stigma scale adapted from Macq and colleagues (Macq et al., 2006), translated into Amharic, and piloted (Ambaw et al., 2015). In this sample, the scale had an $\alpha$ value of 0.84 and a mean interitem correlation coefficient of 0.34 . Higher scores on the TB-related stigma scale show a higher level of stigma.

(IV) Substance use: alcohol, tobacco, and khat use were measured using the WHO Alcohol, Smoking and Substance Involvement Screening Test (ASSIST) (version 3.1) (WHO, 2010). The ASSIST was designed for use across different cultural settings. The instrument's psychometric properties have been tested using data from multiple countries, including low, middle, and high income countries and shown to be valid, reliable, and easy to administer across settings (Humeniuk et al., 2008). The ASSIST risk score ranges from 0-31 for tobacco and 0-39 for alcohol and khat. The risk score of the respondents obtained for alcohol is categorized into 'low' (0 to 10), 'moderate' (11 to 26 ) or 'high' risk (above 26), and for khat low (0 to 3), moderate (4 to 26), and high (above 26) (WHO, 2010).

(V) Comorbid illness: data on the presence of chronic illnesses other than TB were obtained by asking the question 'Have you ever been told by health professionals to have cardiac illness, hypertension, diabetes mellitus, depression, or mental illnesses other than depression?' HIV status was recorded from the TB register after informed consent.

(VI) Type of TB: It refers to whether the TB was pulmonary or extra-pulmonary. It was captured from the TB register in the health facilities using a structured checklist.

\section{Recruitment and ethics}

People with newly diagnosed TB who fulfilled the inclusion criteria were identified, informed, and invited to participate in the study by health professionals running TB clinics at the health facilities. When the individuals expressed interest to participate, they were linked to trained nurse research assistants who provided them detail information, sought written informed consent or witnessed thumb print, and carried out the data collection generally at the health facilities. The information provided to participants was explained face-to-face and delivered in written form to participants. The proposal was approved by the Institutional Review Board of College of Health Sciences of Addis Ababa University (number 027/14/Psy) before data collection. In the process of data collection, respondents who endorsed the suicide item of PHQ-9 were referred to health workers within the health facilities for further evaluation and treatment.

\section{Follow up}

The first (Baseline) assessment was done at the time of starting the medications. The second ( 2 months): assessment was done after participants took anti-TB medication for 2 months. The third (6 month) assessment was done after participants took the full course of their anti-TB medication. Figure 1 details the flow of participants at each assessment time.

\section{Ascertainment of time to follow up}

Time of loss to follow-up was taken as midway between the last successful attempt to contact and the first unsuccessful attempt to contact, following the convention when exact times are not captured (Rothman et al., 2008). Participants assessed at baseline but unavailable for measurements at 2 months were defined as contributing one person-month. Participants assessed at 2 months but unavailable at 6 months were defined as contributing four person-months. Participants followed for the whole 6 months had six person-months.

\section{Data analysis}

Data were analyzed using STATA version 15 (StataCorp. URL: http://www.stata.com). Descriptive statistics were used to summarize the data and to calculate incidence rates of depression. Poisson regression was used to identify predictors of incidence and findings were presented using adjusted $\beta$ (beta) coefficients (Lumley and Kronmal, 2006). Robust and cluster standard errors were used as the study institutions were located in the southern and northern regions of the country. The inclusion of independent variables in the multivariable analysis was based on its theoretical importance and adequacy of the number of participants in cells for each category (Tabachnic and Fidell, 2007). Regression analysis included participants with more than one assessment for depression. Statistical significance was set at $p$ value less than 0.05 . The Strengthening the Reporting of Observational Studies in Epidemiology guidelines have been used to report our findings (von Elm et al., 2007).

\section{Results}

\section{Characteristics of participants}

A total of 648 participants were assessed at baseline for depression. Sixty two (9.6\%) transferred out of the study area after being recruited into the study. Participants were in the age range of $18-85$ years with a median of 30 years. Just over half $(n=348 ; 53.7 \%)$ were male, $224(34.6 \%)$ had no formal education, and $172(26.5 \%)$ were farmers. The average annual household 
Total number of people with TB who registered at the primary care facilities for TB care during the study period $(n=965)$

Excluded $(n=304)$

1. Under age $(n=145)$

2. Plan for transfer out $(n=21)$

3. Taken anti-TB drugs for $>1$ month $(n=31)$

4. Admitted for $>5$ days over the last 1 month $(n=30)$

5. Confirmed to have MDR-TB $(n=11)$

6. On retreatment regimen $(n=57)$

7. Excluded because they did not speak the language $(\mathrm{N}=9)$

Refusal $(n=4)$

Total number of people with TB recruited at baseline into the study ( $n=657)$

Left the study before the second month $(n=63)$

1. Treatment default $(n=6)$

2. Death $(n=22)$

3. Refused $(n=3)$

4. Transferred out $(n=25$

5. Diagnosis changed $(n=7)$

Total number of people with TB measured at the second month $(n=594)$

Left the study before the sixth month $(n=62)$

1. Treatment default $(n=8)$

2. Death $(n=7)$

3. Refusal $(n=8)$

4. Transferred out $(n=37)$

5. Diagnosis changed $(n=2)$

Total number of people with TB measured at the sixth month $(n=532)$

Fig. 1. Flow chart of participants of the study.

income was 659.0 USD (standard deviation =697.0) (Table 1). Seventy four participants (11.4\%) were living with HIV. Five, three, and one participant had diabetes, cardiac illness, and hypertension respectively. No participant reported to have a previously diagnosed depression. Twelve (1.9\%), eleven (1.7\%), and four $(0.6 \%)$ had high risk alcohol, khat, and tobacco use respectively (Table 2).

\section{Incidence rate of depression}

Two hundred ninety nine participants without baseline depression were followed for a total of 299 and 1076 person-months at the 2 nd month and the end of the follow up respectively. Two hundred seventy nine participants had more than one assessment for depression. The median duration between starting ant-TB medication and the first (baseline) assessment was zero days with a mean of 1.6 days and a standard deviation of 3.5 days. The second (2 months) assessment was done after participants took anti-TB medication for a median duration of 56.0 days with a mean of 57.0 days and a standard deviation of
3.0 days. The third ( 6 month) assessment was done after participants took their anti-TB medication for a median duration of 160 days with a mean of 162 days and a standard deviation of 4.8 days.

Among the 299 participants who did not have probable depression at baseline, $22(7.4 \%)$ developed within the first 2 months of follows up; other four participants developed probable depression in the next 4 months making the total number of new cases $26(8.7 \%)$ (Fig. 2). The incidence rates of depression at 2 and 6 months were 73.6 (95\% CI 42.8-104.3) and $24.2(95 \%$ CI 14.9-33.5) per 1000 person-months, respectively. At 2 months, being female (adjusted $\beta=0.40 ; 95 \%$ CI $0.16-0.63$ ), married (adjusted $\beta=0.37 ; 95 \%$ CI $0.34-0.40$ ), and having pulmonary TB $v$. extra-pulmonary TB (adjusted $\beta=0.23$; 95\% CI $0.001-$ 0.45 ) independently predicted incidence. Higher perceived social support was found to be protective against depression onset (adjusted $\beta=-0.15 ; 95 \%$ CI -0.23 to -0.07 ). At 6 months, these variables were still independent predictors of incidence except marital status (adjusted $\beta=0.04 ; 95 \% \mathrm{CI}-0.001$ to 0.08 ) (Table 3). 
Table 1. Socio-demographic characteristics of participants $(n=648)$

\begin{tabular}{|c|c|}
\hline Variable & Number $(\%) /$ mean; range \\
\hline \multicolumn{2}{|l|}{ Sex } \\
\hline Male & $348(53.7)$ \\
\hline Female & $300(46.30)$ \\
\hline Age in years & $34.4 \pm 14.6 ; 18-85$ \\
\hline \multicolumn{2}{|l|}{ Marital status } \\
\hline Single & $210(32.4)$ \\
\hline Married & $358(55.3)$ \\
\hline Widowed or divorced & $80(12.4)$ \\
\hline \multicolumn{2}{|l|}{ Level of education } \\
\hline No formal education & $224(34.6)$ \\
\hline Primary education & $260(40.1)$ \\
\hline Secondary or above & $164(25.3)$ \\
\hline \multicolumn{2}{|l|}{ Occupation } \\
\hline Unemployed & $37(5.7)$ \\
\hline Government employee & $61(9.4)$ \\
\hline Self-employed & $133(20.5)$ \\
\hline Farmer & $172(26.5)$ \\
\hline Student & $39(6.0)$ \\
\hline Housewife & $111(17.1)$ \\
\hline Daily laborer & $44(6.8)$ \\
\hline Other & $51(7.9)$ \\
\hline Annual household income in USD & $659.0 \pm 697.0 ; 24.0-5940.0$ \\
\hline \multicolumn{2}{|l|}{ Religion } \\
\hline Christian & $429(66.2)$ \\
\hline Muslim & $219(33.8)$ \\
\hline \multicolumn{2}{|l|}{ Residence } \\
\hline Urban & $364(56.2)$ \\
\hline Rural & $284(43.8)$ \\
\hline \multicolumn{2}{|l|}{ Ethnicity } \\
\hline Amhara & $306(47.2)$ \\
\hline Gurage & $192(29.6)$ \\
\hline Mareko & $68(10.5)$ \\
\hline Silte & $65(10.0)$ \\
\hline Oromo & $11(1.7)$ \\
\hline Other & $6(0.9)$ \\
\hline $\begin{array}{l}\text { Perceived social support (Oslo-3 } \\
\text { score) }\end{array}$ & $9.8 \pm 2.5 ; 3-14$ \\
\hline TB stigma score & $27.0 \pm 7.6 ; 10-48$ \\
\hline
\end{tabular}

\section{Discussion}

A high incidence rate of probable depression was found in people with newly diagnosed TB undergoing treatment using the DOTS regimen. In a previous manuscript we found that depression was associated with increased mortality and treatment default rates in people with TB (Ambaw et al., 2018). In general, depression
Table 2. Illness and substance use in the participants $(N=648)$

\begin{tabular}{|c|c|}
\hline Variables & Number (\%) \\
\hline \multicolumn{2}{|l|}{ Type of TB } \\
\hline Pulmonary & $371(57.3)$ \\
\hline Extra pulmonary & $277(42.8)$ \\
\hline \multicolumn{2}{|l|}{ HIV status } \\
\hline Negative & $495(76.4)$ \\
\hline Positive & 74 (11.4) \\
\hline Unknown & $79(12.2)$ \\
\hline \multicolumn{2}{|l|}{ Hyper tension } \\
\hline No & $647(99.8)$ \\
\hline Yes & $1(0.2)$ \\
\hline \multicolumn{2}{|l|}{ Cardiac illness } \\
\hline No & $645(99.5)$ \\
\hline Yes & $3(0.5)$ \\
\hline \multicolumn{2}{|l|}{ Diabetes mellitus } \\
\hline No & $643(99.2)$ \\
\hline Yes & $5(0.8)$ \\
\hline \multicolumn{2}{|l|}{ Prior depression } \\
\hline No & $648(100)$ \\
\hline Yes & 0 \\
\hline \multicolumn{2}{|l|}{ Level of alcohol risk: } \\
\hline Low & $562(86.7)$ \\
\hline Moderate & 74 (11.4) \\
\hline High & $12(1.9)$ \\
\hline \multicolumn{2}{|l|}{ Level of tobacco risk } \\
\hline Low & $615(94.9)$ \\
\hline Moderate & $29(4.5)$ \\
\hline High & $4(0.6)$ \\
\hline \multicolumn{2}{|l|}{ Level of khat risk } \\
\hline Low & $544(84.0)$ \\
\hline Moderate & $93(14.3)$ \\
\hline High & $11(1.7)$ \\
\hline
\end{tabular}

co-occurring with physical conditions decreases treatment adherence (Katon, 2011) and substantially increases mortality (Wulsin et al., 1999). Taking the harm comorbid depression in people with TB can cause (Sweetland et al., 2017; Ambaw et al., 2018), and the fact that the incident cases occurred in people who were under the attention of healthcare providers into consideration, the incidence rate observed in our study can be interpreted as alarmingly high. In fact, the incidence rate we found is higher than what was reported for people living with HIV both in Uganda, Africa (Kinyanda et al., 2016) and France, Europe (Nacher et al., 2010; Elenga et al., 2014), type-2 diabetic patients (Lunghi et al., 2016), or nursing homes (Boorsma et al., 2012). Previous researchers also reported that depression is more prevalent in people with TB than with other physical illnesses (Doherty et al., 2013). The global incidence rate of unipolar depression 


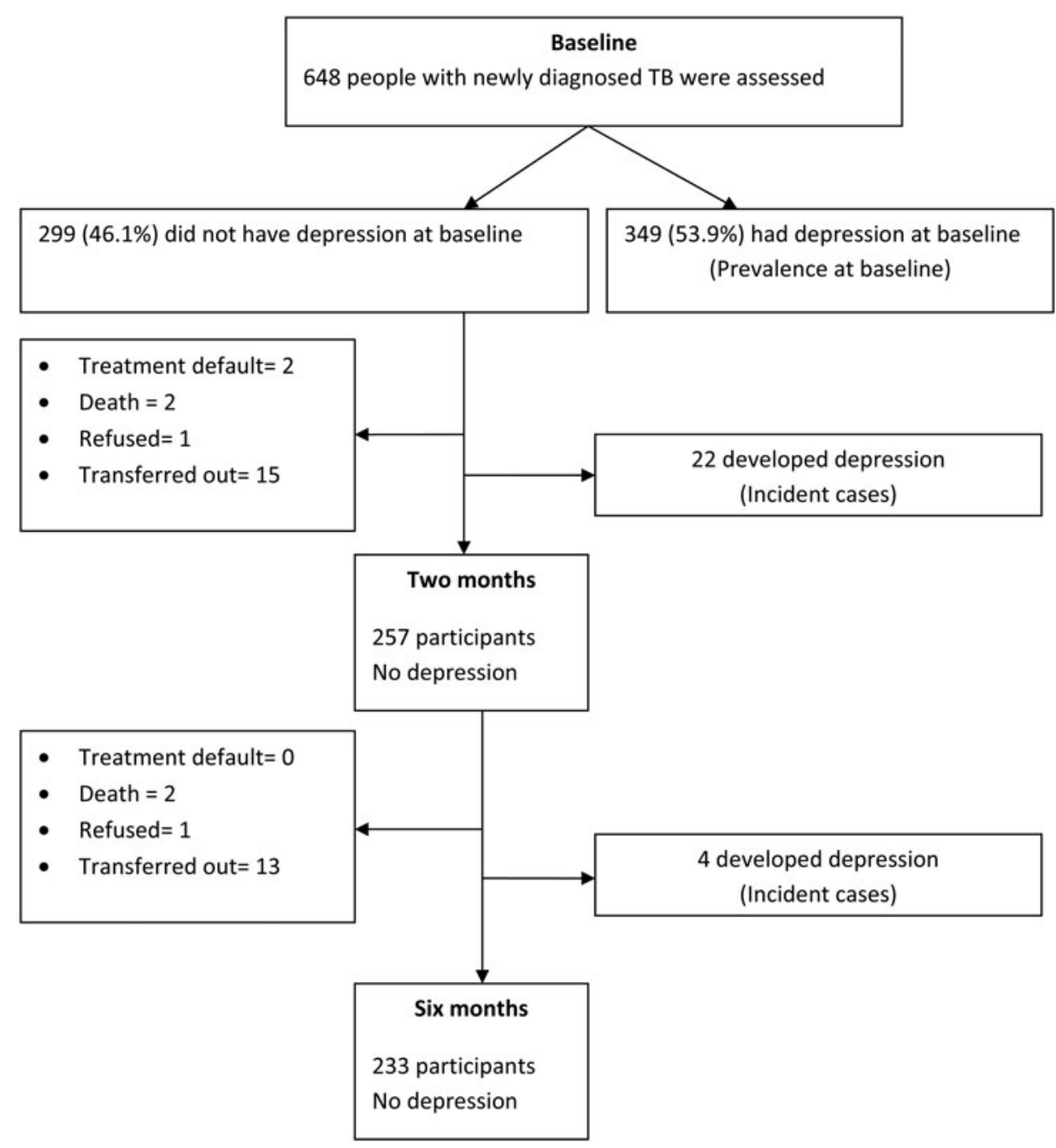

Fig. 2. Flow chart of follow up of participants who did not have baseline probable depression.

was estimated to be $49 / 100000 /$ year for women and 31/100000/ year for men in 2000 (Ustun et al., 2004), incomparably lower than that observed in TB suggesting that TB care guidelines should include mental health components. Sweetland and colleagues (Sweetland et al., 2018) have proposed that TB care is an appropriate component of the existing health services to integrate mental health in primary care in low-resource settings.

Higher incidence of depression in people with TB compared to the general population had been reported in Taiwan (Shen et al., 2014, Yen et al., 2015). The incidence rate observed in our study was substantially higher than that reported in Taiwan probably because studies in Taiwan followed people with TB for about 12 years including mainly TB-free period of the participants (Shen et al., 2014; Yen et al., 2015). Shen et al. (2014) found the highest number of incident cases during the first 6 months which seems to agree with our finding.

New onset depression in people with newly diagnosed TB could be the result of the active inflammatory process directly leading to depression in susceptible individuals (Herbert and Cohen, 1993; Raison et al., 2006; Dantzer et al., 2008), stress of encountering an infectious diseases with risk to others, decreased functioning from illness and psychological loss (Pachi et al., 2013), and, taking multiple drugs with warnings that failure to adhere will have dire consequences. The first 2 months from the start of anti-TB medications is also a period when most medication side effects occur
(Yee et al., 2003; Gulbay et al., 2006). With time, people with TB are more likely to develop better coping strategies (Olley et al., 2006). The relative reduction in the incidence rate after 2 months in the presence of continued isoniazid and discontinued ethambutol doses may be a point of interest for future investigation on the relationship of these medications with depression.

Our finding that female sex predicts incidence of depression agrees with previous studies in various populations (Kessler et al., 1993; Buchtemann et al., 2012; Huang et al., 2012; Nefs et al., 2012; Elenga et al., 2014; Lunghi et al., 2016). Likewise, the protective effect of social support was in agreement with what was observed in diabetes cohort (Lunghi et al., 2016). This finding can be a supportive evidence to assert the notion that enhancing the support system of TB patients can reduce depression. The higher incidence of depression in people with pulmonary TB than for extra pulmonary TB may be related to the higher communicability of pulmonary TB which puts all close contacts, mainly household members, at a high risk of developing $\mathrm{TB}$, and this undue cognitive stress could lead to depression; but biological mechanisms should also be investigated.

\section{Limitations}

We used a screening tool to assess depression. We might have reported undiagnosed prevalent cases as incident as the health 
Table 3. Predictors of incidence of depression in TB patients $(n=279)$

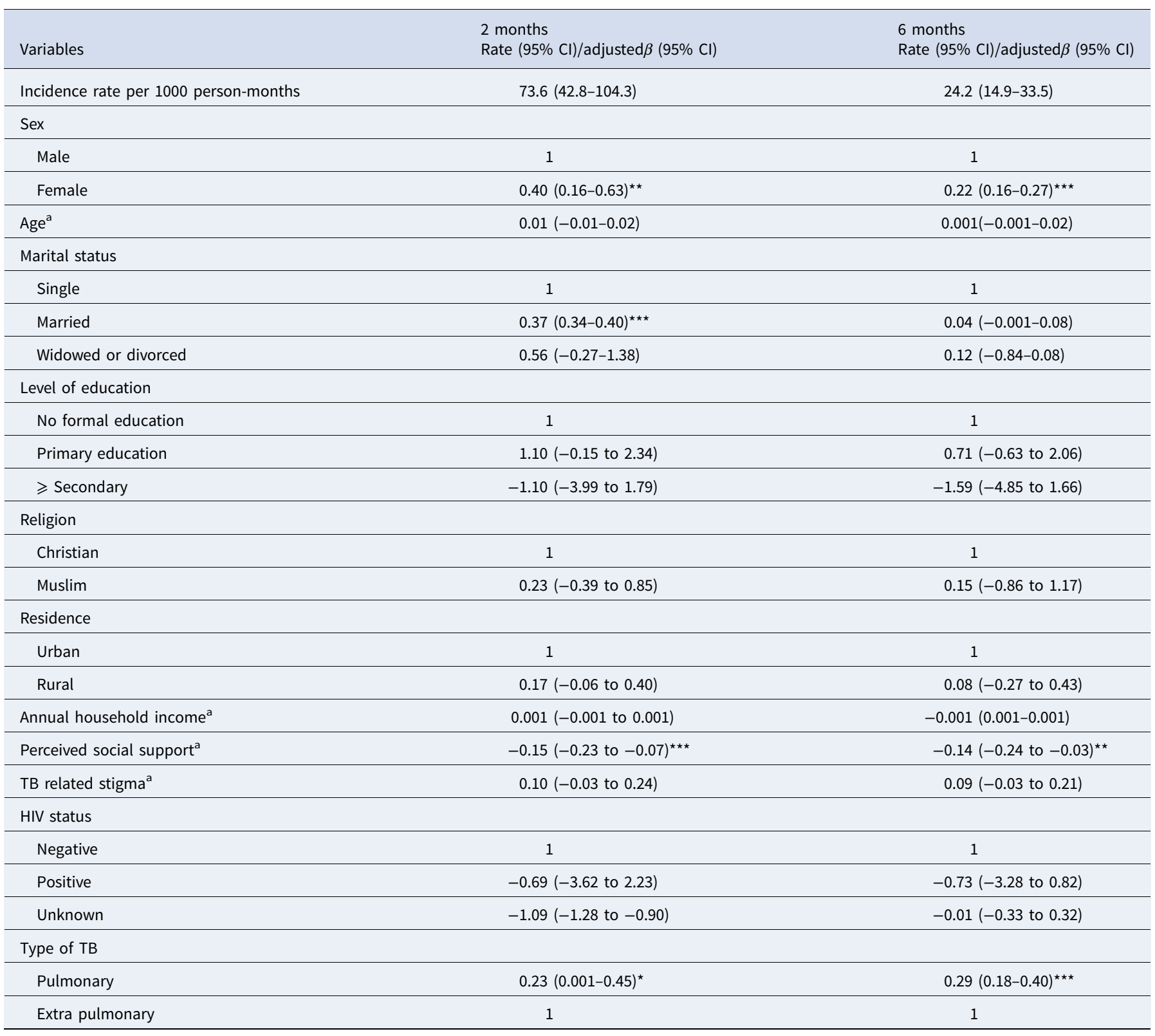

Adjusted $\beta=$ regression coefficients; ${ }^{\star}=p<0.05 ;{ }^{\star \star}=p<0.01 ;{ }^{\star \star \star \star \star}=p<0.001$

Adjustment was done for all the variables in the table.

${ }^{a}$ The variables were measured at the continuous level.

system may not be strong enough to diagnose depression in the context of TB where there is overlap between $\mathrm{TB}$ and depression symptoms. For example, none of the participants who scored above the cut point of PHQ-9 and none of the participants who we referred to health professionals because they endorsed the 'suicide item of PHQ-9' were diagnosed to have depression by the trained primary care workers.

Undiagnosed physical illnesses, poverty, and loss to follow up might have confounded our findings. Because the study was conducted in a setting where the health system is not as such strong, our participants could have had undiagnosed comorbid physical illnesses that might have increased incidence of depression. Another potentially confounding factor is poverty which may not have been fully captured by our socio-demographic variables. Lastly, we had no information on whether participants transferred out of the study area differed significantly from others in terms of developing depression. In addition to that our conclusions cannot be extended to TB patients who are hospitalized, are being re-treated, or have multidrug-resistant disease.

Our sampling technique was a family of non-probability sampling. Nevertheless, as the consecutive sample met criteria for robustness (multiple sites, long data collection period, all eligible participants approached to participate) (Mathieson, 2014) it should be reasonably representative of adults with newly diagnosed $\mathrm{TB}$ in the outpatient department. Theoretically, information bias was a possibility due to a change in the capacity of the assessors as they become more familiar with more practice over time. There are no statistical methods to check this bias and its direction cannot be determined in this specific situation. However, the rigorous training given to 
field workers before starting data collection is expected to minimize this bias.

A potential concern regarding generalizability is homogeneity of the TB treatment approach in other settings and the sustainability of the current practice of $\mathrm{TB}$ treatment in future (Partridge et al., 2015). TB is being treated according to the DOTS approach introduced by the WHO in 1994 (Onozaki and Raviglione, 2010) and this approach is being adhered in the global strategy of 'End-TB by 2030' (World Health Organization, 2015). It is therefore similar across low-income country settings and is likely to be sustained in the coming years. In conclusion, the observed findings would seem to be reasonably valid for the study population as well as being generalizable to adults with newly diagnosed TB in outpatient settings elsewhere in Ethiopia as well as in similar settings in Sub Saharan Africa.

\section{Conclusions}

The high incidence rate of probable depression was found in people with newly diagnosed TB and undergoing treatment. Most incident cases occurred during the first 2 months and the incidence rate relatively decreased with time during the continuation phase of anti-TB treatment despite continued use of isoniazid. The long term course of incident cases of depression, and for how long incidence rate remains high in this population after the TB is treated requires further study. TB care guidelines should have mental health components.

Acknowledgements. We are grateful to our research participants, research assistants, and officials of health institutions.

Author contributions. Fa, RM, $\mathrm{CH}$, and AA contributed to the design and undertaking of the study. FA trained research assistances, supervised field work, and drafted the manuscript. All authors reviewed the manuscript and approved the final version.

Financial support. The research was mainly funded by the European Union, Seventh Framework Programme (FP7/2007-2013) under the Emerald grant agreement number 305968, and partially funded by the Psychiatry Research Trust. The funding sources had no involvement in the conduct of the research or in the preparation of the article. FA and $\mathrm{CH}$ receive support from AMARI (African Mental Health Research Initiative), which is funded through the DELTAS Africa Initiative (DEL-15-01). The DELTAS Africa Initiative is an independent funding scheme of the African Academy of Sciences (AAS)'s Alliance for Accelerating Excellence in Science in Africa (AESA) and supported by the New Partnership for Africa's Development Planning and Coordinating Agency (NEPAD Agency) with funding from the Wellcome Trust (DEL-15-01) and the UK government. The views expressed in this publication are those of the author(s) and not necessarily those of AAS, NEPAD Agency, Wellcome Trust or the UK government. $\mathrm{CH}$ is supported by the National Institute of Health Research (NIHR) Global Health Research Unit on Health System Strengthening in Sub-Saharan Africa, King's College London (GHRU 16/136/54). The views expressed are those of the author(s) and not necessarily those of the NHS, the NIHR or the Department of Health and Social Care.

Conflict of interest. None.

Ethical standards. The authors assert that all procedures contributing to this work comply with the ethical standards of the relevant national and institutional committees on human experimentation and with the Helsinki Declaration of 1975, as revised in 2008.

\section{References}

Ambaw F, Mayston R, Hanlon C and Alem A (2015) Depression among patients with tuberculosis: determinants, course and impact on pathways to care and treatment outcomes in a primary care setting in southern Ethiopia - a study protocol. BMJ Open 5, e007653.

Ambaw F, Mayston R, Hanlon C and Alem A (2017) Burden and presentation of depression among newly diagnosed individuals with TB in primary care settings in Ethiopia. BMC Psychiatry 17, 57.

Ambaw F, Mayston R, Hanlon C, Medhin G and Alem A (2018) Untreated depression and tuberculosis treatment outcomes, quality of life and disability, Ethiopia. Bulletin of the World Health Organization 96, 243-255.

Aydin IO and Ulusahin A (2001) Depression, anxiety comorbidity, and disability in tuberculosis and chronic obstructive pulmonary disease patients: applicability of GHQ-12. General Hospital Psychiatry 23, 77-83.

Boorsma M, Joling K, Dussel M, Ribbe M, Frijters D, van Marwijk HW, Nijpels G and van Hout $\mathbf{H}$ (2012) The incidence of depression and its risk factors in Dutch nursing homes and residential care homes. The American Journal of Geriatric Psychiatry 20, 932-942.

Buchtemann D, Luppa M, Bramesfeld A and Riedel-Heller S (2012) Incidence of late-life depression: a systematic review. Journal of Affective Disorders 142, 172-179.

Dantzer R, o'Connor JC, Freund GG, Johnson RW and Kelley KW (2008) From inflammation to sickness and depression: when the immune system subjugates the brain. Nature Reviews Neuroscience 9, 46-56.

Doherty AM, Kelly J, McDonald C, O’Dywer AM, Keane J and Cooney J (2013) A review of the interplay between tuberculosis and mental health. General Hospital Psychiatry 35, 398-406.

Duko B, Gebeyehu A and Ayano G (2015) Prevalence and correlates of depression and anxiety among patients with tuberculosis at WolaitaSodo University Hospital and Sodo Health Center, WolaitaSodo, South Ethiopia, Cross sectional study. BMC Psychiatry 15, 015-0598.

Elenga N, Georger-Sow MT, Messiaen T, Lamaury I, Favre I, Nacher M and Beaucaire G (2014) Incidence and predictive factors of depression among patients with HIV infection in Guadeloupe: 1988-2009. International Journal of STD \& AIDS 25, 559-563.

Federal Ministry of Health of Ethiopia (2012a) Guidelines for Clinical and Programmatic Managment of TB, Leprosy and TB/HIV in Ethiopia, 5th Edn. Addis Ababa: Falcon Printing.

Federal Ministry of Health of Ethiopia (2012b) Ethiopian mental health strategy. Available at http://www.globalmentalhealth.org/sites/default/files/ Ethiopia\%20MH\%20Strategy.pdf (Accessed 26 December 2018).

Gelaye B, Williams MA, Lemma S, Deyessa N, Bahretibeb Y, Shibre T, Wondimagegn D, Lemenhe A, Fann JR, Vander Stoep A and Andrew Zhou XH (2013) Validity of the patient health questionnaire-9 for depression screening and diagnosis in East Africa. Psychiatry Research 210, 653-661.

Gulbay BE, Gurkan OU, Yildiz OA, Onen ZP, Erkekol FO, Baccioglu A and Acican T (2006) Side effects due to primary antituberculosis drugs during the initial phase of therapy in 1149 hospitalized patients for tuberculosis. Respiratory Medicine 100, 1834-1842.

Hanlon C, Medhin G, Selamu M, Breuer E, Worku B, Hailemariam M, Lund C, Prince M and Fekadu A (2015) Validity of brief screening questionnaires to detect depression in primary care in Ethiopia. Journal of Affective Disorders 186, 32-39.

Herbert TB and Cohen S (1993) Depression and immunity: a meta-analytic review. Psychological Bulletin 113, 472-486.

Huang CJ, Lin CH, Lee MH, Chang KP and Chiu HC (2012) Prevalence and incidence of diagnosed depression disorders in patients with diabetes: a national population-based cohort study. General Hospital Psychiatry 34, 242-248.

Humeniuk R, Ali R, Babor TF, Farrell M, Formigoni ML, Jittiwutikarn J, de Lacerda RB, Ling W, Marsden J, Monteiro M, Nhiwatiwa S, Pal H, Poznyak V and Simon S (2008) Validation of the Alcohol, Smoking And Substance Involvement Screening Test (ASSIST). Addiction 103 1039-1047.

Ige OM and Lasebikan VO (2011) Prevalence of depression in tuberculosis patients in comparison with non-tuberculosis family contacts visiting the DOTS clinic in a Nigerian tertiary care hospital and its correlation with disease pattern. Mental Health in Family Medicine 8, 235-241.

Issa BA, Yussuf AD and Kuranga SI (2009) Depression comorbidity among patients with tuberculosis in a university teaching hospital outpatient clinic in Nigeria. Mental Health in Family Medicine 6, 133-138. 
Katon WJ (2011) Epidemiology and treatment of depression in patients with chronic medical illness. Dialogues in Clinical Neuroscience 13, 7-23.

Katon W, Lin EH and Kroenke K (2007) The association of depression and anxiety with medical symptom burden in patients with chronic medical illness. General Hospital Psychiatry 29, 147-155.

Kessler RC, McGonagle KA, Swartz M, Blazer DG and Nelson CB (1993) Sex and depression in the National Comorbidity Survey. I: lifetime prevalence, chronicity and recurrence. Journal of Affective Disorders 29, 85-96.

Kiecolt-Glaser JK and Glaser R (2002) Depression and immune function: central pathways to morbidity and mortality. Journal of Psychosomatic Research 53, 873-876.

Kinyanda E, Weiss HA, Levin J, Nakasujja N, Birabwa H, Nakku J, Mpango R, Grosskurth H, Seedat S, Araya R and Patel V (2016) Incidence and persistence of major depressive disorder among people living with HIV in Uganda. AIDS and Behavior 21, 1641-1654.

Kroenke K, Spitzer RL, Williams JB and Lowe B (2010) The patient health questionnaire somatic, anxiety, and depressive symptom scales: a systematic review. General Hospital Psychiatry 32, 345-359.

Lumley T and Kronmal R (2006) Relative risk regression in medical research: models, contrasts, estimators, and algorithms. Biostatistics working paper series, University of Washington. paper 293. Available at http://www. bepress.com/uwbiostat/paper293 (Accessed 23 May 2016).

Lunghi C, Moisan J, Gregoire JP and Guenette L (2016) Incidence of depression and associated factors in patients with type 2 diabetes in Quebec, Canada: a population-based cohort study. Medicine (Baltimore) 95, e3514.

Macq J, Solis A and Martinez G (2006) Assessing the stigma of tuberculosis. Psychology, Health \& Medicine 11, 346-352.

Madan A, Jain NK, Sharma TN, Sharma DK and Madan R (1989) Attempted suicide following treatment with isoniazid. Tubercle $\mathbf{7 0}$, 147-149.

Mathai PJ, Ravindran P, Joshi P and Sundaram P (1981) Psychiatric morbidity in pulmonary tuberculosis-a clinical study. Indian Journal of Psychiatry 23, 66-68.

Mathieson K (2014) Making sense of biostatistics: types of nonprobability sampling. Journal of Clinical Research Best Practices 10(10). doi: https:// www.magiworld.org/journal/2014/1410 biostatistics64.pdf.

Meltzer H (2003). Development of a common instrument for mental health. In Nosikov A and Gudex C (eds), EUROHIS: Developing Common Instruments for Health Surveys. Amsterdam: IOS Press, pp. 35-50.

Mikkelsen RL, Middelboe T, Pisinger C and Stage KB (2004) Anxiety and depression in patients with chronic onstructive pulmonary disease (COPD). A review. Nordic Journal of Psychiatry 58, 65-70.

Moussas G, Tselebis A, Karkanias A, Stamouli D, Ilias I, Bratis D and Vassila-Demi K (2008) A comparative study of anxiety and depression in patients with bronchial asthma, chronic obstructive pulmonary disease and tuberculosis in a general hospital of chest diseases. Annals of General Psychiatry 7, 7.

Nacher M, Adriouch L, Godard Sebillotte C, Hanf M, Vantilcke V, El Guedj M, Vaz T, Leconte C, Simart G, Djossou ML and Couppie P (2010) Predictive factors and incidence of anxiety and depression in a cohort of HIV-positive patients in French Guiana. AIDS Care 22, 1086-1092.

Nefs G, Pouwer F, Denollet J and Pop V (2012) The course of depressive symptoms in primary care patients with type 2 diabetes: results from the Diabetes, Depression, Type D Personality Zuidoost-Brabant (DiaDDZoB) Study. Diabetologia 55, 608-616.

Olley BO, Seedat S and Stein DJ (2006) Persistence of psychiatric disorders in a cohort of HIV/AIDS patients in South Africa: a 6-month follow-up study. Journal of Psychosomatic Research 61, 479-484.

Onozaki I and Raviglione M (2010) Stopping tuberculosis in the 21st century: goals and strategies. Respirology 15, 32-43.

Pachi A, Bratis D, Moussas G and Tselebis A (2013) Psychiatric morbidity and other factors affecting treatment adherence in pulmonary tuberculosis patients. Tuberculosis Research and Treatment 2013, 489865.
Panchal SL (2011) Correlation with duration and depression in TB patients in rural Jaipur district (NIMS Hospital). International journal of Pharma and Bio Sciences 2, 263-267.

Partridge SR, Juan SJ, McGeechan K, Bauman A and Allman-Farinelli M (2015) Poor quality of external validity reporting limits generalizability of overweight and/or obesity lifestyle prevention interventions in young adults: a systematic review. Obesity Reviews 16, 13-31.

Raison CL, Capuron L and Miller AH (2006) Cytokines sing the blues: inflammation and the pathogenesis of depression. Trends in Immunology 27, 24-31.

Reiche EM, Nunes SO and Morimoto HK (2004) Stress, depression, the immune system, and cancer. The Lancet Oncology 5, 617-625.

Rothman KJ, Greenland S and Lash TL (2008) Modern Epidemiology. USA: Lippincott Williams \& Wilkins.

Shen TC, Wang CY, Lin CL, Liao WC, Chen CH, Tu CY, Hsia TC, Shih CM, Hsu WH and Chung CJ (2014) People with tuberculosis are associated with a subsequent risk of depression. European Journal of Internal Medicine 25, 936-940.

Sulehri MA, Dogar IA, Sohail H, Mehdi Z, Azam M, Niaz O, Javed MS, Sajjad IA and Iqbal Z (2010) Prevalence of depression among tuberculosis patients. A.P.M.C 4, 133-137.

Sweetland AC, Jaramillo E, Wainberg ML, Chowdhary N, Oquendo MA, Medina-Marino A and Dua T (2018) Tuberculosis: an opportunity to integrate mental health services in primary care in low-resource settings. The Lancet Psychiatry 5, 952-954.

Sweetland AC, Kritski A, Oquendo MA, Sublette ME, Norcini Pala A, Silva LRB, Karpati A, Silva EC, Moraes MO, Silva J and Wainberg ML (2017) Addressing the tuberculosis-depression syndemic to end the tuberculosis epidemic. The International Journal of Tuberculosis and Lung Disease 21, 852-861.

Tabachnic BG and Fidell LS (2007) Using Multivariate Statistics. USA: Pearson Educ. Inc.

Trenton AJ and Currier GW (2001) Treatment of comorbid tuberculosis and depression. Primary Care Companion To the Journal of Clinical Psychiatry 3, 236-243.

Ustun TB, Ayuso-Mateos JL, Chatterji S, Mathers C and Murre CJL (2004) Global burden of depressive disorders in the year 2000. British Journal of Psychiatry 184, 386-392.

Vega P, Sweetland A, Acha J, Castillo H, Guerra D, Smith Fawzi MC and Shin S (2004) Psychiatric issues in the management of patients with multidrug-resistant tuberculosis. The International Journal of Tuberculosis and Lung Disease 8, 749-759.

von Elm E, Altman DG, Egger M, Pocock SJ, Gotzsche PC, Vandenbroucke JP and Initiative S (2007) Strengthening the Reporting of Observational Studies in Epidemiology (STROBE) statement: guidelines for reporting observational studies. BMJ 335, 806-808.

WHO (2010) The Alcohol, Smoking and Substance Involvement Screening Test (ASSIST): Manual for use in primary care. Available at http://apps. who.int/iris/bitstream/10665/44320/1/9789241599382_eng.pdf (Accessed 17 March 2015).

WHO (2015) The global plan to end TB 2016-2020: the paradiagm shift.

WHO (2016) Mental health gap action programme intervention guide for mental, neurological and substance use disorders in non-specialized health settings. Version 2.0.

Wulsin LR, Vaillant GE and Wells VE (1999) A systematic review of the mortality of depression. Psychosomatic Medicine 61, 6-17.

Yee D, Valiquette C, Pelletier M, Parisien I, Rocher I and Menzies D (2003) Incidence of serious side effects from first-line antituberculosis drugs among patients treated for active tuberculosis. American Journal of Respiratory and Critical Care Medicine 167, 1472-1477.

Yen YF, Chung MS, Hu HY, Lai YJ, Huang LY, Lin YS, Chou P and Deng CY (2015) Association of pulmonary tuberculosis and ethambutol with incident depressive disorder: a nationwide, population-based cohort study. Journal of Clinical Psychiatry 76, e505-e511. 